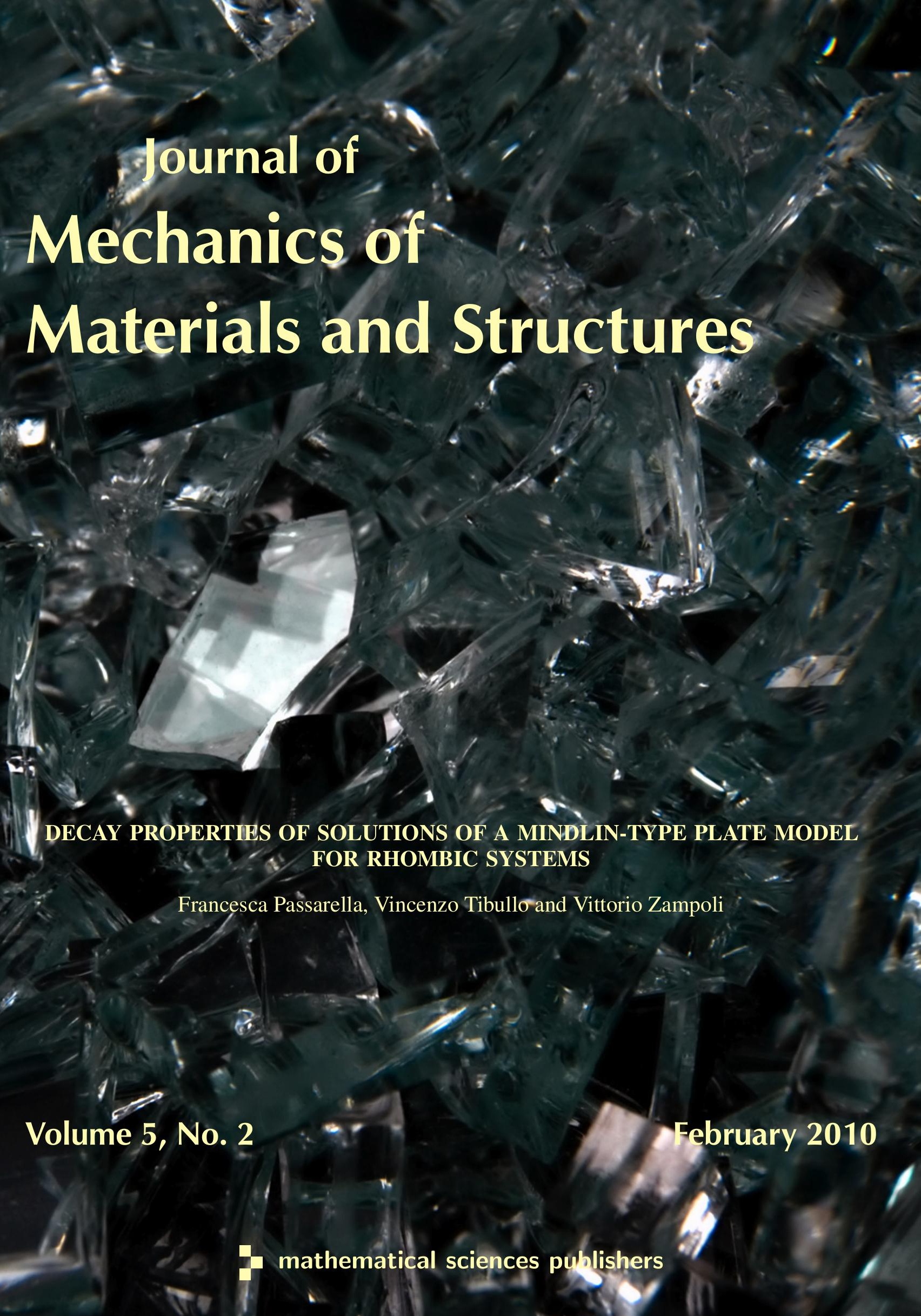




\title{
DECAY PROPERTIES OF SOLUTIONS OF A MINDLIN-TYPE PLATE MODEL FOR RHOMBIC SYSTEMS
}

\author{
Francesca Passarella, Vincenzo Tibullo And VitTorio Zampoli
}

\begin{abstract}
In the present paper, we investigate the spatial behavior of transient and steady-state solutions for the problem of bending applied to a linear Mindlin-type plate model; the plate is supposed to be made of a material characterized by rhombic isotropy, with the elasticity tensor satisfying the strong ellipticity condition. First, using an appropriate family of measures, we show that the transient solution vanishes at distances greater than $c T$ from the support of the given data on the time interval $[0, T]$, where $c$ is a characteristic material constant. For distances from the support less than $c T$, we obtain a spatial decay estimate of Saint-Venant type. Then, for a plate whose middle section is modelled as a (bounded or semiinfinite) strip, a family of measures is used to obtain an estimate describing the spatial behavior of the amplitude of harmonic vibrations, provided that the frequency is lower than a critical value.
\end{abstract}

\section{A list of symbols can be found on page 337.}

\section{Introduction}

Mechanical structures involving elastic plates are useful in a wide range of technical applications and have been the subject of many studies, such as [Green and Naghdi 1967; Naghdi 1971; Lagnese and Lions 1988]. Initially, authors considered the plate model based on Kirchhoff's elastic strain-displacement relations, completely neglecting the effects of transverse shear forces [Nowinski 1978; Lagnese and Lions 1988]. Then, increasingly refined models were introduced, taking into account not only the deflection of the middle section, but also transverse shear deformations. The theory of elastic plates based on the Mindlin model has been developed by Constanda [1990] for the elastostatic bending of a thin slab, including the effects of transverse shear deformation. Furthermore, a dynamic model for small deformations of a thin thermoelastic plate was developed by Schiavone and Tait [1993].

The Reissner-Mindlin and Kirchhoff-Love models are the two most common models of a thin linear elastic plate. It is often remarked in the engineering literature that the Reissner-Mindlin model is more accurate, particularly for thin plates and when transverse shear strain plays a significant role [Hughes 1987]; in fact, both Mindlin [1951] and Reissner [1945; 1947] independently proposed theories which also include the effects of transverse shear deformation. Arnold et al. [2002] showed that the ReissnerMindlin plate bending model has a wider range of applicability than the Kirchhoff-Love model. Under the assumption of constant body force density in the transverse direction, they proved that the ReissnerMindlin model solution converges to the three-dimensional linearly elastic solution in the relative energy norm for the full range of surface loads. Fabrizio and Chiriță [2004] studied the deformations of a linear viscoelastic plate, including transverse shear deformations.

Keywords: plates, rhombic systems, strong ellipticity, transient and steady-state solutions.

The work in this paper was performed under the auspices of the GNFM of the Italian INdAM.. 
The state of bending for a transversely isotropic plate of Mindlin-type has been analyzed by Passarella and Zampoli [2009a; 2009b]. The first of these papers contains a uniqueness result and a Galerkin representation of the solution, without positive definiteness assumptions on the elasticity tensor. Furthermore, under the hypotheses of positive definiteness of the elasticity tensor, the authors prove a Gurtin type variational theorem and a minimum principle. In [Passarella and Zampoli 2009b], previous hypotheses are relaxed and, assuming strong ellipticity of the elasticity tensor, results about the spatial behavior of transient and steady-state solutions are established.

With regard to the spatial behavior of solutions, Saint-Venant's principle plays a central role in the theory and applications of elasticity. A comprehensive review of research on the spatial behavior of solutions is given by [Toupin 1965; Knowles 1966; Flavin 1974; Horgan and Knowles 1983; Gregory and Wan 1985; Horgan 1989; 1996; Mielke 1988].

For genuine dynamic problems of elasticity, useful information on the spatial behavior of solutions can be furnished by the well-known domain of influence theorem as presented by Gurtin [1972] and ascribed to Wheeler and Sternberg [1968]. According to Gurtin the domain of influence of the given external data at time $T$ consists of the set $D_{T}$ of all points in the body that can be reached by signals propagating from the support $\widehat{D}_{T}$, on the time interval [0,T], with speeds equal to or less than the maximum speed of propagation $c$. Then the domain of influence theorem shows that on $[0, T]$ the externally given data have no effect on points outside of $D_{T}$.

In the present paper we study a bending state of a rhombic system modelled as a (either bounded or unbounded) Mindlin-type plate, under a strong ellipticity condition on the elasticity tensor. In this context, we use the properties of rhombic systems widely investigated by Gurtin [1972] and Chiriță et al. [2007]. Such systems, together with transversely isotropic materials, show a good applicability to mathematical models of plates that take into account transverse shear deformations (see [Paroni et al. 2006] for details). In particular, in the context of the linear theory, we investigate the spatial behavior of transient and steady-state solutions. This investigation is concerned with some properties of the solutions of the evolution equations ruling the displacement and rotation fields, supposing the plate to be initially at rest. Moreover, time-dependent displacements and rotation fields are imposed on its boundary. These fields on the boundary induce disturbances that propagate in the interior of the plate.

Outline of paper. In Section 2, we derive the evolution equations for the two-dimensional theory and state assumptions concerning the strong ellipticity of the elasticity tensor.

In Section 3, considering the spatial support of data contained in a bounded domain $D_{T}^{*}$, we define a set of appropriate line-integral measures $\Phi_{\kappa}$ and establish some estimates in order to obtain the domain of influence and the spatial decay of transient solutions away from $D_{T}^{*}$. Using these estimates and following the time-weighted power function method [Chiriță and Ciarletta 1999; 2003; Ciarletta et al. 2005], we determine the speed of propagation of mechanical disturbances from $D_{T}^{*}$, as well as the spatial decay of solutions.

In Section 4, we treat a more specific mechanical problem, concerning the steady-state vibrations of a (either bounded or semiinfinite) strip. Starting from an idea of Ciarletta et al. [2005] and Chiriță [1995], we introduce a set of appropriate line-integral measures $\mathscr{F}_{\kappa}$ associated with the amplitude of timeharmonic vibrations. We obtain a differential inequality describing the behavior of steady-state solutions under the hypothesis that the frequency of harmonic vibrations is lower than a certain critical value. 


\section{Formulation of the problem}

In this paper, we study the behavior of a homogeneous body filled by a material that occupies at time $t=0$ a right cylinder $\bar{B}$ of height $2 h$ with (bounded or unbounded) cross-section $\bar{\Sigma}$ and smooth lateral boundary $\Pi$. We call $B$ and $\Sigma$ the interiors of $\bar{B}$ and $\bar{\Sigma}$; we choose the rectangular Cartesian coordinate frame in such a way that $O x_{1} x_{2}$ is the middle plane of the cylinder and thus its faces are located at $x_{3}= \pm h$. We also suppose that $\Sigma$ is a simply connected region, $\partial \Sigma$ is the boundary of $\Sigma$, and $h \ll \operatorname{diam} \Sigma$. Latin subscripts (unless otherwise specified) range over the integers $\{1,2,3\}$, whereas Greek subscripts are confined to the range $\{1,2\}$; summation over repeated subscripts is implied. Superposed dots, or subscripts preceded by a comma, mean a partial derivative with respect to time or to the corresponding Cartesian variables. When needed, vector fields are represented by bold-type letters. We will disregard regularity questions, simply assuming a degree of smoothness sufficient to ensure analysis is valid.

For each point $\left(x_{1}, x_{2}, x_{3}\right) \in \bar{B}$ and $t \in[0, \infty)$, a bending state for such an elastic cylinder is characterized by

$$
u_{\alpha}\left(x_{1}, x_{2}, x_{3}, t\right)=-u_{\alpha}\left(x_{1}, x_{2},-x_{3}, t\right) \quad \text { and } \quad u_{3}\left(x_{1}, x_{2}, x_{3}, t\right)=u_{3}\left(x_{1}, x_{2},-x_{3}, t\right),
$$

where $u_{i}$ are the components of the displacement vector that vary smoothly with respect to $x_{3}$. Nevertheless, as with the study carried out in [Passarella and Zampoli 2009a; 2009b], we restrict our attention to the bending state characterized by

$$
u_{\alpha}\left(x_{1}, x_{2}, x_{3}, t\right)=x_{3} v_{\alpha}\left(x_{1}, x_{2}, t\right) \quad \text { and } \quad u_{3}\left(x_{1}, x_{2}, x_{3}, t\right)=w\left(x_{1}, x_{2}, t\right),
$$

and we assume that the components $f_{i}$ of the body force vector obey the relations

$$
f_{\alpha}\left(x_{1}, x_{2}, x_{3}, t\right)=-f_{\alpha}\left(x_{1}, x_{2},-x_{3}, t\right), \quad f_{3}\left(x_{1}, x_{2}, x_{3}, t\right)=f_{3}\left(x_{1}, x_{2},-x_{3}, t\right) .
$$

The behavior of thin plates of uniform thickness is described by the equations of motion

$$
\frac{1}{3} h^{2} M_{\beta \alpha, \beta}-\tau_{\alpha 3}+H_{\alpha}=\frac{1}{3} h^{2} \varrho \ddot{v}_{\alpha}, \quad \tau_{\beta 3, \beta}+F=\varrho \ddot{w}, \quad \text { on } \bar{\Sigma} \times(0, \infty)
$$

and the constitutive equations

$$
\begin{aligned}
M_{i j}\left(x_{1}, x_{2}, t\right) & =\frac{3}{2 h^{3}} \int_{-h}^{h} x_{3} C_{i j k l} u_{k, l}\left(x_{1}, x_{2}, x_{3}, t\right) d x_{3}, \\
\tau_{i j}\left(x_{1}, x_{2}, t\right) & =\frac{1}{2 h} \int_{-h}^{h} C_{i j k l} u_{k, l}\left(x_{1}, x_{2}, x_{3}, t\right) d x_{3},
\end{aligned}
$$

where $\varrho$ is the reference mass density, $C_{i j k l}$ are the components of the elasticity tensor obeying the usual symmetry relations, and

$$
\begin{gathered}
F\left(x_{1}, x_{2}, t\right)=\frac{1}{2 h} \int_{-h}^{h} f_{3}\left(x_{1}, x_{2}, x_{3}, t\right) d x_{3}+\frac{1}{h} C_{33 k l} u_{k, l}\left(x_{1}, x_{2}, h, t\right), \\
H_{\alpha}\left(x_{1}, x_{2}, t\right)=\frac{1}{2 h} \int_{-h}^{h} x_{3} f_{\alpha}\left(x_{1}, x_{2}, x_{3}, t\right) d x_{3}+C_{3 \alpha k l} u_{k, l}\left(x_{1}, x_{2}, h, t\right) .
\end{gathered}
$$

Suppose that

$$
H_{\alpha}=0, \quad F=0, \quad \text { on } \bar{\Sigma} \times[0, \infty) .
$$


In what follows, we consider a homogeneous rhombic elastic material, with the group $\mathscr{C}_{3}$ generated $^{1}$ by $\boldsymbol{R}_{\boldsymbol{e}_{3}}^{\pi}$ and $\boldsymbol{R}_{\boldsymbol{e}_{2}}^{\pi}$ characterized by (see, for example, [Gurtin 1972])

$$
\begin{aligned}
& C_{1123}=C_{1131}=C_{1112}=C_{2223}=C_{2231}=C_{2212}=0, \\
& C_{3323}=C_{3331}=C_{3312}=C_{2331}=C_{2312}=C_{3112}=0 .
\end{aligned}
$$

Using these equations and defining as usual

$$
\begin{aligned}
& c_{11}=C_{1111}, \quad c_{22}=C_{2222}, \quad c_{33}=C_{3333}, \quad c_{44}=C_{2323}, \quad c_{55}=C_{1313}, \\
& c_{66}=C_{1212}, \quad c_{12}=C_{1122}, \quad c_{23}=C_{2233}, \quad c_{31}=C_{3311},
\end{aligned}
$$

the constitutive equations (2-2) for rhombic materials become

$$
\begin{gathered}
M_{11}=c_{11} v_{1,1}+c_{12} v_{2,2}, \quad M_{22}=c_{12} v_{1,1}+c_{22} v_{2,2}, \quad M_{12}=M_{21}=c_{66}\left(v_{1,2}+v_{2,1}\right), \\
\tau_{13}=\tau_{31}=c_{55}\left(w_{, 1}+v_{1}\right), \quad \tau_{23}=\tau_{32}=c_{44}\left(w_{, 2}+v_{2}\right) .
\end{gathered}
$$

Now, we restrict our attention to the class of rhombic materials having a strongly elliptic elasticity tensor, that is,

$$
C_{i j k l} m_{i} m_{k} n_{j} n_{l}>0
$$

for all nonzero vectors $\left(m_{1}, m_{2}, m_{3}\right)$ and $\left(n_{1}, n_{2}, n_{3}\right)$. As it is possible to see in [Gurtin 1972], this hypothesis on the elasticity tensor is weaker than positive definiteness. Chiriță et al. [2007] prove that for rhombic systems the strong ellipticity condition (2-7) is equivalent to

$$
\begin{aligned}
c_{11} n_{1}^{2} m_{1}^{2}+c_{22} n_{2}^{2} & m_{2}^{2}+c_{33} n_{3}^{2} m_{3}^{2}+c_{66}\left(n_{1} m_{2}+n_{2} m_{1}\right)^{2}+c_{44}\left(n_{3} m_{2}+n_{2} m_{3}\right)^{2} \\
& +c_{55}\left(n_{1} m_{3}+n_{3} m_{1}\right)^{2}+2 c_{12} n_{1} m_{1} n_{2} m_{2}+2 c_{23} n_{2} m_{2} n_{3} m_{3}+2 c_{31} n_{3} m_{3} n_{1} m_{1}>0
\end{aligned}
$$

and this relation implies the conditions

$$
\begin{aligned}
& c_{11}>0, \quad c_{22}>0, \quad c_{33}>0, \quad c_{44}>0, \quad c_{55}>0, \quad c_{66}>0, \\
& \left|c_{12}+c_{66}\right|<c_{66}+\sqrt{c_{11} c_{22}}, \quad\left|c_{13}+c_{55}\right|<c_{55}+\sqrt{c_{11} c_{33}}, \quad\left|c_{23}+c_{44}\right|<c_{44}+\sqrt{c_{22} c_{33}} \text {. }
\end{aligned}
$$

\section{Transient solutions: decay estimate of Saint-Venant type}

In this section, we establish results describing the spatial behavior of solutions of the given data on the interval $[0, T]$ under the strong ellipticity condition on the elasticity tensor. To this end, we can rewrite the system of (2-1), (2-3), (2-5), and (2-6) in terms of $\left(v_{\alpha}, w\right)$ as

$$
\begin{aligned}
\frac{1}{3} h^{2}\left[c_{11} v_{1,11}+c_{66} v_{1,22}+\left(c_{12}+c_{66}\right) v_{2,12}\right]-c_{55}\left(w_{, 1}+v_{1}\right) & =\frac{1}{3} h^{2} \varrho \ddot{v}_{1}, \\
\frac{1}{3} h^{2}\left[c_{66} v_{2,11}+c_{22} v_{2,22}+\left(c_{12}+c_{66}\right) v_{1,12}\right]-c_{44}\left(w_{, 2}+v_{2}\right) & =\frac{1}{3} h^{2} \varrho \ddot{v}_{2}, \\
c_{55}\left(w_{, 11}+v_{1,1}\right)+c_{44}\left(w_{, 22}+v_{2,2}\right) & =\varrho \ddot{w},
\end{aligned}
$$

or, equivalently, as

$$
\frac{1}{3} h^{2} \widetilde{M}_{\beta \alpha, \beta}-\tau_{\alpha 3}=\frac{1}{3} \varrho h^{2} \ddot{v}_{\alpha}, \quad \tau_{\beta 3, \beta}=\varrho \ddot{w},
$$

\footnotetext{
${ }^{1} \boldsymbol{R}_{\boldsymbol{u}}^{\theta}$ is the orthogonal tensor corresponding to a right-handed rotation through the angle $\theta$ about an axis of unit vector $\boldsymbol{u}$.
} 
where $\widetilde{M}_{\beta \alpha}$ represents the family of tensors depending on the parameter $\kappa$ through

$$
\begin{array}{ll}
\widetilde{M}_{11}=c_{11} v_{1,1}+\left(c_{66}-\kappa\right) v_{2,2}, & \widetilde{M}_{12}=\left(c_{12}+\kappa\right) v_{1,2}+c_{66} v_{2,1}, \\
\widetilde{M}_{21}=c_{66} v_{1,2}+\left(c_{12}+\kappa\right) v_{2,1}, & \widetilde{M}_{22}=\left(c_{66}-\kappa\right) v_{1,1}+c_{22} v_{2,2},
\end{array}
$$

and $\tau_{\beta 3}$ is defined by Equation (2-6). The family of tensors $\widetilde{M}_{\beta \alpha}$ has a nonvanishing skew-symmetric part, depending on the skew-symmetric part of $v_{\alpha, \beta}$.

Let $\mathscr{P}$ be the problem defined by (2-6), (3-2), (3-3) and the initial-boundary conditions

$$
\begin{aligned}
v_{\alpha}\left(x_{1}, x_{2}, 0\right) & =0, & w\left(x_{1}, x_{2}, 0\right) & =0 & & \text { on } \Sigma, \\
\dot{v}_{\alpha}\left(x_{1}, x_{2}, 0\right) & =0, & \dot{w}\left(x_{1}, x_{2}, 0\right) & =0 & & \text { on } \Sigma, \\
v_{\alpha} & =\breve{v}_{\alpha}, & w & =\breve{w} & & \text { on } L \times[0,+\infty), \\
v_{\alpha} & =0, & w & =0 & & \text { on } L_{C} \times[0,+\infty),
\end{aligned}
$$

where $L$ and $L_{C}$ are disjoint and complementary nonempty subsets of $\partial \Sigma$ and $\mathscr{D}=\left(\breve{v}_{\alpha}, \breve{w}\right)$ is the set of assigned fields representing the external data of the problem in question.

We define the support $\widehat{D}_{T}$ of external data $\mathscr{D}$ on the time interval $[0, T]$ as the set of points

$$
\boldsymbol{x}=\left(x_{1}, x_{2}\right) \in L
$$

for which there exists $\tau \in[0, T]$ such that $\breve{v}_{\alpha}(\boldsymbol{x}, \tau) \neq 0$ or $\breve{w}(\boldsymbol{x}, \tau) \neq 0$. In other words, for $\boldsymbol{x} \in \partial \Sigma \backslash \widehat{D}_{T}$, we have

$$
\breve{v}_{\alpha}(\boldsymbol{x}, t)=0 \quad \text { and } \quad \breve{w}(\boldsymbol{x}, t)=0 \quad \text { for all } t \in[0, T] .
$$

For convenience, we assume that $\widehat{D}_{T}$ is a nonempty bounded set and that $D_{T}^{*}$ is the smallest regular subcurve of $L$ including $\widehat{D}_{T}$. We consider the sets

$$
D_{r}=\left\{x \in \bar{\Sigma}: D_{T}^{*} \cap S(x, r) \neq \varnothing\right\}, \quad \Sigma_{r}=\bar{\Sigma} \backslash D_{r}, \quad \Sigma\left(r_{1}, r_{2}\right)=\Sigma_{r_{2}} \backslash \Sigma_{r_{1}},
$$

where $r>0, r_{2} \leq r_{1}$, and $S(\boldsymbol{x}, r)$ is the closed ball with radius $r$ and center at $\boldsymbol{x}$, that is,

$$
S(\boldsymbol{x}, r)=\left\{\boldsymbol{y} \in \mathbb{R}^{2}:|\boldsymbol{y}-\boldsymbol{x}| \leq r\right\} .
$$

Then, $L_{r}=\partial \Sigma_{r} \cap \Sigma$ is the subcurve of $\partial \Sigma_{r}$ that lies inside the inner part of $\bar{\Sigma}$, and whose unit normal vector $\boldsymbol{n}$ is directed towards the interior of $\Sigma_{r}$ (or, equivalently, towards the exterior of $D_{r}$ ).

We also agree that, for $r=0, D_{0}$ and $L_{0}$ coincide with $D_{T}^{*}$, and the normal vector $\boldsymbol{n}$ to $L_{0}$ is directed towards the interior of $\Sigma$.

For any positive parameter $\lambda$, we introduce the function

$$
\Phi_{\kappa}(r, t)=-\int_{0}^{t} \int_{L_{r}} e^{-\lambda s}\left[\tau_{\beta 3}(s) \dot{w}(s)+\frac{1}{3} h^{2} \widetilde{M}_{\beta \alpha}(s) \dot{v}_{\alpha}(s)\right] n_{\beta} d l d s, \quad r \geq 0, \quad t \in[0, T] ;
$$

for what follows, it is useful to remark that

$$
\Phi_{\kappa}(r, 0)=0 \quad \text { for all } r \geq 0 .
$$


From direct differentiation of (3-5) with respect to the variable $t$, we get

$$
\frac{\partial \Phi_{\kappa}}{\partial t}(r, t)=-\int_{L_{r}} e^{-\lambda t}\left[\tau_{\beta 3}(t) \dot{w}(t)+\frac{1}{3} h^{2} \widetilde{M}_{\beta \alpha}(t) \dot{v}_{\alpha}(t)\right] n_{\beta} d l .
$$

On the other hand, from the definition of $\Sigma_{r}$, from (3-4), and from the divergence theorem, we have

$$
\Phi_{\kappa}\left(r_{1}, t\right)-\Phi_{\kappa}\left(r_{2}, t\right)=-\int_{0}^{t} \int_{\Sigma\left(r_{1}, r_{2}\right)} e^{-\lambda s}\left[\tau_{\beta 3}(s) \dot{w}(s)+\frac{1}{3} h^{2} \widetilde{M}_{\beta \alpha}(s) \dot{v}_{\alpha}(s)\right]_{, \beta} d \sigma d s .
$$

Using (2-6) and (3-2)-(3-5), we obtain, for $r_{2}<r_{1}$,

$$
\begin{array}{r}
\Phi_{\kappa}\left(r_{1}, t\right)-\Phi_{\kappa}\left(r_{2}, t\right) \\
=-\frac{1}{2} \int_{0}^{t} \int_{\Sigma\left(r_{1}, r_{2}\right)} e^{-\lambda s} \frac{\partial}{\partial s}\left\{\varrho \left[\dot{w}^{2}(s)\right.\right. \\
\left.+\frac{1}{3} h^{2} \dot{v}_{\alpha}(s) \dot{v}_{\alpha}(s)\right]+c_{55}\left(w_{, 1}(s)+v_{1}(s)\right)^{2}+c_{44}\left(w, 2(s)+v_{2}(s)\right)^{2} \\
+\frac{1}{3} h^{2}\left[c_{11} v_{1,1}^{2}(s)+c_{22} v_{2,2}^{2}(s)+2\left(c_{66}-\kappa\right) v_{1,1}(s) v_{2,2}(s)\right. \\
+ \\
\left.\left.+c_{66}\left(v_{2,1}^{2}(s)+v_{1,2}^{2}(s)\right)+2\left(c_{12}+\kappa\right) v_{2,1}(s) v_{1,2}(s)\right]\right\} d \sigma d s .
\end{array}
$$

Introducing

$$
\mathcal{T}=\frac{1}{2} \varrho\left(\dot{w}^{2}+\frac{1}{3} h^{2} \dot{v}_{\alpha} \dot{v}_{\alpha}\right), \quad \mathscr{W}=\frac{1}{2}\left(\mathcal{W}_{0}+\frac{1}{3} h^{2} W_{1}+\frac{1}{3} h^{2} W_{2}\right),
$$

and

$$
\begin{aligned}
& \mathscr{W}_{0}=c_{55}\left(w_{, 1}+v_{1}\right)^{2}+c_{44}\left(w_{, 2}+v_{2}\right)^{2}, \quad \mathscr{W}_{1}=c_{11} v_{1,1}^{2}+c_{22} v_{2,2}^{2}+2\left(c_{66}-\kappa\right) v_{1,1} v_{2,2}, \\
& \mathscr{W}_{2}=c_{66}\left(v_{2,1}^{2}+v_{1,2}^{2}\right)+2\left(c_{12}+\kappa\right) v_{2,1} v_{1,2},
\end{aligned}
$$

it follows from (3-8) that

$$
\Phi_{\kappa}\left(r_{1}, t\right)-\Phi_{\kappa}\left(r_{2}, t\right)=-\int_{0}^{t} \int_{\Sigma\left(r_{1}, r_{2}\right)} e^{-\lambda s} \frac{\partial}{\partial s}[\mathcal{T}(s)+\mathscr{W}(s)] d \sigma d s \quad \text { if } r_{2}<r_{1} .
$$

Now, it is possible to show that $\Phi_{\kappa}$ is continuously differentiable with respect to $r$; in fact, considering

$$
\begin{aligned}
\lim _{r_{1} \rightarrow r_{2}} \frac{\Phi_{\kappa}\left(r_{1}, t\right)-\Phi_{\kappa}\left(r_{2}, t\right)}{r_{1}-r_{2}} & =-\lim _{r_{1} \rightarrow r_{2}} \frac{1}{r_{1}-r_{2}} \int_{0}^{t} \int_{\Sigma\left(r_{1}, r_{2}\right)} e^{-\lambda s} \frac{\partial}{\partial s}[\mathcal{T}(s)+\mathscr{W}(s)] d \sigma d s \\
& =-\int_{0}^{t} \int_{L_{r}} e^{-\lambda s} \frac{\partial}{\partial s}[\mathcal{T}(s)+\mathcal{W}(s)] d \sigma d s,
\end{aligned}
$$

and integrating by parts with respect to the time variable $s$, we arrive at

$$
\frac{\partial \mathscr{I}_{\kappa}}{\partial r}(r, t)=-\int_{L_{r}} e^{-\lambda t}[\mathscr{T}(t)+\mathscr{W}(t)] d l-\lambda \int_{0}^{t} \int_{L_{r}} e^{-\lambda s}[\mathscr{T}(s)+\mathscr{W}(s)] d l d s .
$$

Let $\mathscr{A}_{0}, \mathscr{A}_{1}$, and $\mathscr{A}_{2}$ be the matrices associated with the quadratic forms $\mathscr{W}_{0}, \mathscr{W}_{1}$, and $\mathscr{W}_{2}$, respectively. When the strong ellipticity condition (2-8) is valid and $\kappa$ satisfies the relation

$$
\max \left\{-c_{66}-c_{12}, c_{66}-\sqrt{c_{11} c_{22}}\right\}<\kappa<\min \left\{c_{66}-c_{12}, c_{66}+\sqrt{c_{11} c_{22}}\right\},
$$

the eigenvalues of $\mathscr{A}_{0}, \mathscr{A}_{1}$, and $\mathscr{A}_{2}$, which are

$$
c_{55}, \quad c_{44}, \quad \frac{1}{2}\left[c_{11}+c_{22} \pm \sqrt{\left(c_{11}-c_{22}\right)^{2}+4\left(c_{66}-\kappa\right)^{2}}\right], \quad c_{66} \pm\left|c_{12}+\kappa\right|,
$$


are all strictly positive. In what follows, we denote with $\mu_{\kappa}$ and $\eta_{\kappa}$ the smallest and largest eigenvalues of $\mathscr{A}_{0}, \mathscr{A}_{1}$, and $\mathscr{A}_{2}$, respectively. These are

$$
\begin{aligned}
& \mu_{\kappa}=\min \left\{c_{44}, c_{55}, \frac{1}{2}\left[c_{11}+c_{22}-\sqrt{\left(c_{11}-c_{22}\right)^{2}+4\left(c_{66}-\kappa\right)^{2}}\right], c_{66}-\left|c_{12}+\kappa\right|\right\}, \\
& \eta_{\kappa}=\max \left\{c_{44}, c_{55}, \frac{1}{2}\left[c_{11}+c_{22}+\sqrt{\left(c_{11}-c_{22}\right)^{2}+4\left(c_{66}-\kappa\right)^{2}}\right], c_{66}+\left|c_{12}+\kappa\right|\right\} .
\end{aligned}
$$

If $\varrho>0$ and (2-8) and (3-13) hold, then $\mathscr{T}$ and $\mathcal{W}$ are positive definite; thus, we show through (3-12) that $\Phi_{\kappa}(r, t)$ is a nonincreasing function with respect to $r$, that is,

$$
\mathscr{I}_{\kappa}\left(r_{1}, t\right) \leq \mathscr{I}_{\kappa}\left(r_{2}, t\right) \quad \text { if } r_{2}<r_{1} .
$$

If the plate is bounded and $\ell=\max _{x \in \bar{\Sigma}}\left\{\min _{\boldsymbol{y} \in D_{T}^{*}} \sqrt{\left(x_{1}-y_{1}\right)^{2}+\left(x_{2}-y_{2}\right)^{2}}\right\}$, we obtain from the definition of $L_{r}$ that $L_{\ell}=\varnothing$ and, consequently,

$$
\Phi_{\kappa}(\ell, t)=0, \quad t \in[0, T]
$$

moreover, (3-11), (3-14), and (3-15) imply that

$$
0 \leq \mathscr{I}_{\kappa}(r, t)=\int_{0}^{t} \int_{\Sigma(\ell, r)} e^{-\lambda s} \frac{\partial}{\partial s}[\mathscr{T}(s)+\mathcal{W}(s)] d \sigma d s \quad \text { for } r \leq \ell .
$$

In this case, it is obvious that $\Sigma(\ell, r)=\Sigma_{r}$. It is possible to obtain a relation similar to (3-16) for an unbounded plate. To this end, we estimate $\mathscr{I}_{\kappa}(r, t)$ and $\partial \Phi_{\kappa}(r, t) / \partial t$ in terms of $\partial \Phi_{\kappa}(r, t) / \partial r$.

Theorem 3.1. Let $\varrho>0$ and suppose the hypotheses (2-8) and (3-13) hold. Let $\left(v_{\alpha}, w\right)$ be a solution of the initial-boundary value problem $\mathscr{P}$ and $\widehat{D}_{T}$ the bounded support of external data $\mathscr{D}$ on the time interval $[0, T]$, then the function $\mathscr{I}_{\kappa}$ satisfies the first-order differential inequalities

$$
\left|\Phi_{\kappa}(r, t)\right|+\frac{c_{\kappa}}{\lambda} \frac{\partial \Phi_{\kappa}}{\partial r}(r, t) \leq 0, \quad\left|\frac{\partial \Phi_{\kappa}}{\partial t}(r, t)\right|+c_{\kappa} \frac{\partial \Phi_{\kappa}}{\partial r}(r, t) \leq 0, \quad \text { with } c_{\kappa}=\sqrt{\frac{\eta_{\kappa}}{\varrho}} .
$$

Proof. Under the hypotheses (2-8) and (3-13), we can observe that

$$
0 \leq k_{m}^{(\alpha)}\left(\gamma_{1}^{2}+\gamma_{2}^{2}\right) \leq \mathscr{F}\left[\mathscr{A}_{\alpha} ; \boldsymbol{\gamma}, \boldsymbol{\gamma}\right] \leq k_{M}^{(\alpha)}\left(\gamma_{1}^{2}+\gamma_{2}^{2}\right)
$$

where $k_{m}^{(\alpha)}$ and $k_{M}^{(\alpha)}$ are the smallest and largest eigenvalues of $\mathcal{W}_{\alpha}$, the functional $\mathscr{F}$ is given by

$$
\mathscr{F}\left[\mathscr{A}_{\alpha} ; \varphi, \gamma\right]:=\varphi \cdot \mathscr{A}_{\alpha} \gamma,
$$

with the variables $\boldsymbol{\varphi}=\left(\varphi_{1}, \varphi_{2}\right)$ and $\boldsymbol{\gamma}=\left(\gamma_{1}, \gamma_{2}\right)$. In particular,

$$
\begin{array}{ll}
k_{m}^{(1)}=\frac{1}{2}\left[c_{11}+c_{22}-\sqrt{\left(c_{11}-c_{22}\right)^{2}+4\left(c_{66}-\kappa\right)^{2}}\right], & k_{m}^{(2)}=c_{66}-\left|c_{12}+\kappa\right|, \\
k_{M}^{(1)}=\frac{1}{2}\left[c_{11}+c_{22}+\sqrt{\left(c_{11}-c_{22}\right)^{2}+4\left(c_{66}-\kappa\right)^{2}}\right], & k_{M}^{(2)}=c_{66}+\left|c_{12}+\kappa\right| .
\end{array}
$$

Through the Schwarz inequality and Equations (3-3) and (3-10), we get

$$
\mathscr{F}\left[\mathscr{A}_{q} ; \boldsymbol{\varphi}, \boldsymbol{\gamma}\right] \leq\left[\mathscr{F}\left[\mathscr{A}_{q} ; \boldsymbol{\varphi}, \boldsymbol{\varphi}\right]\right]^{1 / 2}\left[\mathscr{F}\left[\mathscr{A}_{q} ; \boldsymbol{\gamma}, \boldsymbol{\gamma}\right]\right]^{1 / 2}
$$


and

$$
\begin{array}{lll}
\mathscr{F}\left[\mathscr{A}_{1} ; \hat{\boldsymbol{\gamma}}^{(1)}, \hat{\boldsymbol{\gamma}}^{(1)}\right]=\mathscr{W}_{1}, & & \mathscr{F}\left[\mathscr{A}_{1} ; \widetilde{\boldsymbol{M}}^{(1)}, \hat{\boldsymbol{\gamma}}^{(1)}\right]=\widetilde{M}_{11}^{2}+\widetilde{M}_{22}^{2}, \\
\mathscr{F}\left[\mathscr{A}_{2} ; \hat{\boldsymbol{\gamma}}^{(2)}, \hat{\boldsymbol{\gamma}}^{(2)}\right]=W_{2}, & & \mathscr{F}\left[\mathscr{A}_{2} ; \widetilde{\boldsymbol{M}}^{(2)}, \hat{\boldsymbol{\gamma}}^{(2)}\right]=\widetilde{M}_{21}^{2}+\widetilde{M}_{12}^{2},
\end{array}
$$

where

$$
\hat{\boldsymbol{\gamma}}^{(1)}=\left(v_{1,1}, v_{2,2}\right), \quad \widetilde{\boldsymbol{M}}^{(1)}=\left(\widetilde{M}_{11}, \widetilde{M}_{22}\right), \quad \hat{\boldsymbol{\gamma}}^{(2)}=\left(v_{1,2}, v_{2,1}\right), \quad \widetilde{\boldsymbol{M}}^{(2)}=\left(\widetilde{M}_{21}, \widetilde{M}_{12}\right) .
$$

Now, collecting (3-10) and (3-18)-(3-20), we deduce that

$$
\begin{aligned}
& \widetilde{M}_{11}^{2}+\widetilde{M}_{22}^{2} \leq \mathscr{F}^{1 / 2}\left[\mathscr{A}_{1} ; \widetilde{\boldsymbol{M}}^{(1)}, \widetilde{\boldsymbol{M}}^{(1)}\right] W_{1}^{1 / 2} \leq\left[k_{M}^{(1)}\left(\widetilde{M}_{11}^{2}+\widetilde{M}_{22}^{2}\right)\right]^{1 / 2} W_{1}^{1 / 2}, \\
& \widetilde{M}_{21}^{2}+\widetilde{M}_{12}^{2} \leq \mathscr{F}^{1 / 2}\left[\mathscr{A}_{2} ; \widetilde{\boldsymbol{M}}^{(2)}, \widetilde{\boldsymbol{M}}^{(2)}\right] W_{2}^{1 / 2} \leq\left[k_{M}^{(2)}\left(\widetilde{M}_{21}^{2}+\widetilde{M}_{12}^{2}\right)\right]^{1 / 2} W_{2}^{1 / 2} .
\end{aligned}
$$

On the other hand, from (2-6) and (3-10) it follows that

$$
0 \leq k_{m}^{(0)} \mathscr{W}_{0} \leq \tau_{\beta 3} \tau_{\beta 3} \leq k_{M}^{(0)} \mathscr{W}_{0},
$$

where $k_{m}^{(0)}=\min \left\{c_{44}, c_{55}\right\}$ and $k_{M}^{(0)}=\max \left\{c_{44}, c_{55}\right\}$. Equations (3-9), (3-21), and (3-22) allow us to show easily that

$$
\tau_{\beta 3} \tau_{\beta 3}+\frac{1}{3} h^{2} \widetilde{M}_{\beta \alpha} \widetilde{M}_{\beta \alpha} \leq k_{M}^{(0)} \mathscr{W}_{0}+\frac{1}{3} h^{2} k_{M}^{(1)} \mathscr{W}_{1}+\frac{1}{3} h^{2} k_{M}^{(2)} \mathscr{W}_{2} \leq 2 \eta_{\kappa} W .
$$

Next, the Cauchy-Schwarz and arithmetic-geometric mean inequalities lead to

$$
\left|\left(\tau_{\beta 3} \dot{w}+\frac{1}{3} h^{2} \widetilde{M}_{\beta \alpha} \dot{v}_{\alpha}\right) n_{\beta}\right| \leq \frac{\varepsilon \varrho}{2}\left(\dot{w}^{2}+\frac{1}{3} h^{2} \dot{v}_{\alpha} \dot{v}_{\alpha}\right)+\frac{1}{2 \varepsilon \varrho}\left(\tau_{\beta 3} \tau_{\beta 3}+\frac{1}{3} h^{2} \widetilde{M}_{\beta \alpha} \widetilde{M}_{\beta \alpha}\right) .
$$

Finally, we use the estimates (3-23) and (3-24) in (3-5) and (3-7) in order to obtain

$$
\begin{gathered}
\left|\mathscr{T}_{\kappa}(r, t)\right| \leq \varepsilon \int_{0}^{t} \int_{L_{r}} e^{-\lambda s}\left[\mathscr{T}(s)+\frac{\eta_{\kappa}}{\varepsilon^{2} \varrho} \mathscr{W}(s)\right] d l d s, \\
\left|\frac{\partial \mathscr{I}_{\kappa}}{\partial t}(r, t)\right| \leq \varepsilon \int_{L_{r}} e^{-\lambda t}\left[\mathscr{T}(t)+\frac{\eta_{\kappa}}{\varepsilon^{2} \varrho} \mathscr{W}(t)\right] d l .
\end{gathered}
$$

Recalling (3-12) and setting $\varepsilon=c_{\kappa}$ in (3-25) and (3-26), we derive the differential inequalities (3-17).

Using Theorem 3.1, a result similar to (3-16) may be shown for an unbounded plate. In this case, the variable $r$ ranges in $[0, \infty)$. Thanks to (3-17) 2 and for any pair $\left(r_{0}, t_{0}\right)$ such that $t_{0} \in[0, T]$ and $r_{0} \geq c_{\kappa} t_{0}$, we can see that the functions $\Phi_{\kappa}\left(r, t_{0}+\left(r-r_{0}\right) / c_{\kappa}\right)$ and $\Phi_{\kappa}\left(r, t_{0}-\left(r-r_{0}\right) / c_{\kappa}\right)$ are nonincreasing with respect to $r$. This feature, together with (3-6), implies

$$
\begin{array}{ll}
\Phi_{\kappa}\left(r_{0}, t_{0}\right) \leq \Phi_{\kappa}\left(r_{0}-c_{\kappa} t_{0}, 0\right)=0, & r_{0} \geq r_{0}-c_{\kappa} t_{0}, \\
\Phi_{\kappa}\left(r_{0}, t_{0}\right) \geq \Phi_{\kappa}\left(r_{0}+c_{\kappa} t_{0}, 0\right)=0, & r_{0}+c_{\kappa} t_{0} \geq r_{0},
\end{array} \Longrightarrow \quad \lim _{r_{0} \rightarrow \infty} \Phi_{\kappa}\left(r_{0}, t_{0}\right)=0 .
$$

Taking into account (3-11), (3-14), and (3-27), it follows that

$$
0 \leq \Phi_{\kappa}(r, t)=\lim _{r_{0} \rightarrow \infty} \int_{0}^{t} \int_{\Sigma\left(r_{0}, r\right)} e^{-\lambda s} \frac{\partial}{\partial s}[\mathcal{T}(s)+\mathscr{W}(s)] d \sigma d s,
$$

and it is obvious that $\lim _{r_{0} \rightarrow \infty} \Sigma\left(r_{0}, r\right)=\Sigma_{r}$.

With the help of (3-16) and (3-28), and integrating by parts with respect to $s$, it is possible to prove, for bounded or unbounded plates, the following theorem. 
Theorem 3.2. Let the hypotheses of Theorem 3.1 be still valid. The function $\mathscr{I}_{\kappa}(r, t)$ is nonnegative and

$$
0 \leq \Phi_{\kappa}(r, t)=\int_{\Sigma_{r}} e^{-\lambda t}[\mathcal{T}(t)+\mathscr{W}(t)] d \sigma+\lambda \int_{0}^{t} \int_{\Sigma_{r}} e^{-\lambda s}[\mathscr{T}(s)+\mathscr{W}(s)] d \sigma d s .
$$

Now, since $\mathscr{I}_{\kappa}(r, t)$ is a nonnegative function, we can write from (3-17) 1

$$
\frac{\partial}{\partial r}\left[\exp \left(\frac{\lambda}{c_{\kappa}} r\right) \mathscr{I}_{\kappa}(r, t)\right] \leq 0 \quad \Longrightarrow \quad \mathscr{I}_{\kappa}(r, t) \leq \exp \left(-\frac{\lambda}{c_{\kappa}} r\right) \mathscr{I}_{\kappa}(0, t)
$$

On the other hand, (3-17) 2 implies that the function $\Phi_{\kappa}\left(c_{\kappa} t, t\right)$ is nonincreasing with respect to $t$. This characteristic, together with (3-6), implies

$$
\Phi_{\kappa}\left(c_{\kappa} t, t\right) \leq \Phi_{\kappa}(0,0)=0, \quad t \geq 0 .
$$

We deduce from (3-14) and (3-29) that

$$
0 \leq \Phi_{\kappa}(r, t) \leq \Phi_{\kappa}\left(c_{\kappa} t, t\right), \quad r \geq c_{\kappa} t
$$

Thus, (3-29)-(3-31) imply

$$
\int_{\Sigma_{r}} e^{-\lambda t}[\mathscr{T}(t)+\mathscr{W}(t)] d \sigma+\lambda \int_{0}^{t} \int_{\Sigma_{r}} e^{-\lambda s}[\mathscr{T}(s)+\mathscr{W}(s)] d \sigma d s=0 \quad \text { for } \quad r \geq c_{\kappa} t .
$$

When $\mathscr{T}$ and $\mathcal{W}$ are positive definite, (3-32) is valid if and only if $\mathscr{T}$ and $\mathcal{W}$ are null in $\Sigma_{r}$ for any $r \geq c_{\kappa} t$.

The results obtained up to now lead us to formulate the following theorem about the spatial decay.

Theorem 3.3. Let the hypotheses of Theorem 3.1 be still valid; for each fixed $t \in[0, T]$, we have

$$
\Phi_{\kappa}(r, t) \leq \exp \left(-\frac{\lambda}{c_{\kappa}} r\right) \Phi_{\kappa}(0, t) \quad \text { for } \quad 0 \leq r<c_{\kappa} t, \quad \Phi_{\kappa}(r, t)=0 \quad \text { for } \quad r \geq c_{\kappa} t .
$$

It is easy to observe that, for $t \in[0, T]$ and for each $r \geq c_{\kappa} t,(3-29)$ and (3-33) 2 lead to

$$
\int_{\Sigma_{r}} e^{-\lambda t}[\mathscr{T}(t)+\mathscr{W}(t)] d \sigma+\lambda \int_{0}^{t} \int_{\Sigma_{r}} e^{-\lambda s}[\mathscr{T}(s)+\mathscr{W}(s)] d \sigma d s=0 .
$$

Consequently, when $\varrho>0$ and $\mathscr{W}$ is positive definite, we obtain $\dot{v}_{\alpha}=\dot{w}=0$ on $\Sigma_{r} \times[0, T]$, and through homogeneous initial conditions, we get

$$
v_{\alpha}=w=0 \quad \text { on } \Sigma_{r} \times[0, T] .
$$

Following [Gurtin 1972], we depict the domain of influence of the externally given data at time $T$ as the set of the points of $\bar{\Sigma}$ that can be reached by signals propagating from the support $\hat{D}_{T}$ on the time interval $[0, T]$, with speeds equal to or less than the maximum speed of propagation

$$
c=\sqrt{\frac{\eta}{\varrho}} \quad \text { with } \quad \eta=\inf _{\kappa} \eta_{\kappa},
$$

where $\kappa$ satisfies the relation (3-13). In fact, for a bounded or unbounded plate, we can show by means of (3-34) that on $[0, T]$ the externally given data have no effect on points outside of $D_{c T}$.

Lemma 3.4 (domain of influence). Let the hypotheses of Theorem 3.1 be still valid and let $\left(v_{\alpha}, w\right)$ be a solution of initial-boundary value problem $\mathscr{P}$. Then, $v_{\alpha}=w=0$, on $\Sigma_{c T} \times[0, T]$. 
We can easily prove the following uniqueness result, valid for a bounded or unbounded plate.

Lemma 3.5 (uniqueness). Let the hypotheses of Theorem 3.1 hold. There exists at most one solution for the initial-boundary value problem $\mathscr{P}$.

Proof. Thanks to the linearity of the problem, we have only to show that the null data imply a null solution. Let $\left(\tilde{v}_{\alpha}, \tilde{w}\right)$ be a solution corresponding to the null data. If we choose $T_{1}>0$, since the set $\hat{D}_{T}=\varnothing$ for each $T \in\left(0, T_{1}\right)$ and the function $\Phi_{\kappa}(r, t)=0$, we can conclude that: $v_{\alpha}=w=0$, on $\bar{\Sigma} \times\left[0, T_{1}\right]$.

\section{Steady-state solutions: decay estimate of Saint-Venant type}

Throughout this section, the cross-section $\bar{\Sigma}$ is a rectangular strip, and the problem of steady-state vibrations is studied assuming that $\left(v_{\alpha}, w\right)$ are separable with respect to space and time variables and that the time dependence is periodic, that is $v_{\alpha}=\operatorname{Re}\left[\zeta_{\alpha}\left(x_{1}, x_{2}\right) e^{i \omega t}\right], w=\operatorname{Re}\left[\psi\left(x_{1}, x_{2}\right) e^{i \omega t}\right]$, where $\operatorname{Re}[f]$ represents the real part of $f, \omega \in \mathbb{R}^{+}$is the prescribed frequency of oscillations, and $\zeta_{\alpha}$ and $\psi$ are complex functions. We choose a Cartesian frame of reference such that the middle section of the plate is defined by $\bar{\Sigma}=\left[0, \ell_{1}\right] \times\left[0, \ell_{2}\right] \subset \mathbb{R}^{2}$, where $\ell_{1}$ and $\ell_{2}$ are some positive constants and $\ell_{1}$ can also tend to infinity. Moreover, we impose prescribed harmonic vibrations on the end of the strip located at $x_{1}=0$.

Using the equations of motion (3-1), we conclude that the amplitude $\left(\zeta_{\alpha}, \psi\right)$ satisfies

$$
\begin{aligned}
\frac{1}{3} h^{2}\left[c_{11} \zeta_{1,11}+c_{66} \zeta_{1,22}+\left(c_{12}+c_{66}\right) \zeta_{2,12}\right]-c_{55}\left(\psi_{, 1}+\zeta_{1}\right)+\varrho \frac{1}{3} h^{2} \omega^{2} \zeta_{1} & =0, \\
\frac{1}{3} h^{2}\left[c_{66} \zeta_{2,11}+c_{22} \zeta_{2,22}+\left(c_{12}+c_{66}\right) \zeta_{1,12}\right]-c_{44}\left(\psi_{, 2}+v_{2}\right)+\varrho \frac{1}{3} h^{2} \omega^{2} \zeta_{2} & =0, \\
c_{55}\left(\psi_{11}+\zeta_{1,1}\right)+c_{44}\left(\psi_{, 22}+\zeta_{2,2}\right)+\varrho \omega^{2} \psi & =0,
\end{aligned}
$$

or, equivalently, it satisfies the system

$$
\frac{1}{3} h^{2} \Gamma_{\beta \alpha, \beta}-\chi_{\alpha}+\varrho \frac{1}{3} h^{2} \omega^{2} \zeta_{\alpha}=0, \quad \chi_{\beta, \beta}+\varrho \omega^{2} \psi=0,
$$

with

$$
\begin{aligned}
& \Gamma_{11}=c_{11} \zeta_{1,1}+\left(c_{66}-\kappa\right) \zeta_{2,2}, \quad \Gamma_{12}=\left(c_{12}+\kappa\right) \zeta_{1,2}+c_{66} \zeta_{2,1}, \\
& \Gamma_{21}=c_{66} \zeta_{1,2}+\left(c_{12}+\kappa\right) \zeta_{2,1}, \quad \Gamma_{22}=\left(c_{66}-\kappa\right) \zeta_{1,1}+c_{22} \zeta_{2,2} \text {, }
\end{aligned}
$$

and

$$
\chi_{1}=c_{55}\left(\psi_{, 1}+\zeta_{1}\right), \quad \chi_{2}=c_{44}\left(\psi_{, 2}+\zeta_{2}\right) .
$$

In particular, using (4-1) we can easily show that

$$
\frac{1}{3} h^{2} \Gamma_{1 \alpha, 1}=-\frac{1}{3} h^{2} \Gamma_{2 \alpha, 2}+\chi_{\alpha}-\varrho \frac{1}{3} h^{2} \omega^{2} \zeta_{\alpha}, \quad \chi_{1,1}=-\chi_{2,2}-\varrho \omega^{2} \psi .
$$

Let $\mathscr{P}_{0}$ be the problem defined by (4-1)-(4-3) and by the boundary conditions

$$
\begin{aligned}
& \zeta_{\alpha}\left(x_{1}, 0\right)=0, \quad \psi\left(x_{1}, 0\right)=0, \quad \zeta_{\alpha}\left(x_{1}, \ell_{2}\right)=0, \quad \psi\left(x_{1}, \ell_{2}\right)=0, \quad x_{1} \in\left[0, \ell_{1}\right], \\
& \zeta_{\alpha}\left(\ell_{1}, x_{2}\right)=0, \quad \psi\left(\ell_{1}, x_{2}\right)=0, \quad \zeta_{\alpha}\left(0, x_{2}\right)=\breve{\zeta}_{\alpha}\left(x_{2}\right), \quad \psi\left(0, x_{2}\right)=\breve{\psi}\left(x_{2}\right), \quad x_{2} \in\left[0, \ell_{2}\right],
\end{aligned}
$$

where $\breve{\zeta}_{\alpha}$ and $\breve{\psi}$ are prescribed continuous functions such that

$$
\breve{\zeta}_{\alpha}(0)=0, \quad \breve{\psi}(0)=0, \quad \breve{\zeta}_{\alpha}\left(\ell_{2}\right)=0, \quad \breve{\psi}\left(\ell_{2}\right)=0 .
$$


Using a superposed bar for complex conjugation and defining the segment $L_{x_{1}}=\left\{\left(x_{1}, x_{2}\right): x_{2} \in\left[0, \ell_{2}\right]\right\}$, we introduce the function

$$
\mathscr{F}_{\kappa}\left(x_{1}\right)=\int_{L_{x_{1}}} 2 \operatorname{Re}\left[\bar{\chi}_{1} \psi+\frac{1}{3} h^{2} \bar{\Gamma}_{1 \alpha} \zeta_{\alpha}\right] d x_{2}, \quad x_{1} \in\left[0, \ell_{1}\right] .
$$

Differentiating $\mathscr{F}_{\kappa}$, we obtain

$$
\mathscr{F}_{\kappa}^{\prime}\left(x_{1}\right)=\int_{L_{x_{1}}} 2 \operatorname{Re}\left[\bar{\chi}_{1,1} \psi+\bar{\chi}_{1} \psi_{, 1}+\frac{1}{3} h^{2}\left(\bar{\Gamma}_{1 \alpha, 1} \zeta_{\alpha}+\bar{\Gamma}_{1 \alpha} \zeta_{\alpha, 1}\right)\right] d x_{2} .
$$

Using integration by parts, boundary conditions (4-5), and Equations (4-3), (4-4), and (4-7), we arrive at

$$
\mathscr{F}_{\kappa}^{\prime}\left(x_{1}\right)=\int_{L_{x_{1}}} 2\left[\widehat{\mathscr{W}}-\varrho \omega^{2}\left(\bar{\psi} \psi+\frac{1}{3} h^{2} \bar{\zeta}_{\alpha} \zeta_{\alpha}\right)\right] d x_{2}
$$

where

$$
\begin{aligned}
\widehat{\mathscr{W}} & =\widehat{\mathscr{W}}_{0}+\frac{1}{3} h^{2} \widehat{\mathscr{W}}_{1}+\frac{1}{3} h^{2} \widehat{\mathscr{W}}_{2}, \\
\widehat{\mathscr{W}}_{0} & =c_{55}\left(\bar{\zeta}_{1}+\bar{\psi}_{, 1}\right)\left(\zeta_{1}+\psi, 1\right)+c_{44}\left(\bar{\zeta}_{2}+\bar{\psi}_{, 2}\right)\left(\zeta_{2}+\psi_{, 2}\right), \\
\widehat{\mathscr{W}}_{1} & =c_{11} \bar{\zeta}_{1,1} \zeta_{1,1}+c_{22} \bar{\zeta}_{2,2} \zeta_{2,2}+\left(c_{66}-\kappa\right)\left(\bar{\zeta}_{1,1} \zeta_{2,2}+\zeta_{1,1} \bar{\zeta}_{2,2}\right), \\
\widehat{\mathscr{W}}_{2} & =c_{66}\left(\zeta_{2,1} \bar{\zeta}_{2,1}+\zeta_{1,2} \bar{\zeta}_{1,2}\right)+\left(c_{12}+\kappa\right)\left(\bar{\zeta}_{2,1} \zeta_{1,2}+\zeta_{2,1} \bar{\zeta}_{1,2}\right) .
\end{aligned}
$$

Again, as in the previous section, the matrices associated with $\widehat{\mathscr{W}}_{0}, \widehat{\mathscr{W}}_{1}$, and $\widehat{\mathscr{W}}_{2}$ are $\mathscr{A}_{0}, \mathscr{A}_{1}$, and $\mathscr{A}_{2}$, respectively. When the relations (2-8) and (3-13) are satisfied, we have

$$
\widehat{\mathbb{W}} \geq \mu_{\kappa}\left[\left(\bar{\zeta}_{\alpha}+\bar{\psi}_{, \alpha}\right)\left(\zeta_{\alpha}+\psi_{, \alpha}\right)+\frac{1}{3} h^{2} \bar{\zeta}_{\alpha, \beta} \zeta_{\alpha, \beta}\right]>0 .
$$

Taking into account the well-known membrane problem, we can write

$$
\frac{\pi^{2}}{\ell_{2}^{2}} \int_{L_{x_{1}}} \bar{\zeta}_{\alpha} \zeta_{\alpha} d x_{2} \leq \int_{L_{x_{1}}} \bar{\zeta}_{\alpha, 2} \zeta_{\alpha, 2} d x_{2}, \quad \frac{\pi^{2}}{\ell_{2}^{2}} \int_{L_{x_{1}}} \bar{\psi} \psi d x_{2} \leq \int_{L_{x_{1}}} \bar{\psi}_{, 2} \psi, 2 d x_{2}
$$

and considering (4-8) and (4-9) we arrive at

$$
\mathscr{F}_{\kappa}^{\prime}\left(x_{1}\right) \geq \int_{L_{x_{1}}} 2 \mu_{\kappa}\left[\left(\bar{\zeta}_{\alpha}+\bar{\psi}_{, \alpha}\right)\left(\zeta_{\alpha}+\psi_{, \alpha}\right)+\frac{1}{3} h^{2} \bar{\zeta}_{\alpha, \beta} \zeta_{\alpha, \beta}-\frac{\varrho \omega^{2} \ell_{2}^{2}}{\mu_{\kappa} \pi^{2}}\left(\bar{\psi}_{, 2} \psi_{, 2}+\frac{1}{3} h^{2} \bar{\zeta}_{\alpha, 2} \zeta_{\alpha, 2}\right)\right] d x_{2} .
$$

Using relations (4-10), we can observe that

$$
\frac{6 \ell_{2}^{2}}{\pi^{2} h^{2}} \int_{L_{x_{1}}}\left[\left(\bar{\zeta}_{2}+\bar{\psi}_{, 2}\right)\left(\zeta_{2}+\psi_{, 2}\right)+\frac{1}{6} h^{2} \bar{\zeta}_{2,2} \zeta_{2,2}\right] d x_{2} \geq \frac{\ell_{2}^{2}}{\pi^{2}} \int_{L_{x_{1}}} \zeta_{2,2} \bar{\zeta}_{2,2} d x_{2} \geq \int_{L_{x_{1}}} \zeta_{2} \bar{\zeta}_{2} d x_{2}
$$

On the other side, Schwarz and arithmetic-geometric mean inequalities imply

$$
\left(\bar{\zeta}_{2}+\bar{\psi}_{, 2}\right)\left(\zeta_{2}+\psi_{, 2}\right) \geq(1-\varepsilon) \zeta_{2} \bar{\zeta}_{2}+\left(1-\frac{1}{\varepsilon}\right) \psi_{, 2} \bar{\psi}_{, 2} \quad \text { for every } \varepsilon>0,
$$


and in particular, for $\varepsilon=2,\left(\bar{\zeta}_{2}+\bar{\psi}_{, 2}\right)\left(\zeta_{2}+\psi_{, 2}\right) \geq-\zeta_{2} \bar{\zeta}_{2}+\frac{1}{2} \psi_{, 2} \bar{\psi}_{, 2}$; thus,

$$
\begin{aligned}
\int_{L_{x_{1}}}\left[\left(\bar{\zeta}_{2}+\bar{\psi}_{, 2}\right)\left(\zeta_{2}+\psi, 2\right)+\frac{1}{6} h^{2} \bar{\zeta}_{2,2} \zeta_{2,2}\right] d x_{2} & \geq \int_{L_{x_{1}}}\left(\bar{\zeta}_{2}+\bar{\psi}_{, 2}\right)\left(\zeta_{2}+\psi, 2\right) d x_{2} \\
& \geq \int_{L_{x_{1}}}\left(-\bar{\zeta}_{2} \zeta_{2}+\frac{1}{2} \bar{\psi}_{, 2} \psi_{, 2}\right) d x_{2} .
\end{aligned}
$$

Combining (4-12) and (4-13) we obtain

$$
\int_{L_{x_{1}}}\left[\left(\bar{\zeta}_{2}+\bar{\psi}_{, 2}\right)\left(\zeta_{2}+\psi_{, 2}\right)+\frac{1}{6} h^{2} \bar{\zeta}_{2,2, \zeta_{2,2}}\right] d x_{2} \geq \frac{h^{2} \pi^{2}}{2\left(6 \ell_{2}^{2}+\pi^{2} h^{2}\right)} \int_{L_{x_{1}}} \bar{\psi}_{, 2} \psi_{, 2} d x_{2} .
$$

Moreover, (4-11) and (4-14) imply

$$
\begin{aligned}
\mathscr{F}_{\kappa}^{\prime}\left(x_{1}\right) \geq & \int_{L_{x_{1}}} \mu_{\kappa}\left\{2\left(\bar{\zeta}_{1}+\bar{\psi}_{, 1}\right)\left(\zeta_{1}+\psi_{, 1}\right)+\frac{h^{2} \pi^{2}}{6 \ell_{2}^{2}+\pi^{2} h^{2}}\left[1-\frac{2 \varrho \ell_{2}^{2} \omega^{2}}{\pi^{2} \mu_{\kappa}}\left(1+\frac{6 \ell_{2}^{2}}{\pi^{2} h^{2}}\right)\right] \bar{\psi}_{, 2} \psi_{, 2}\right. \\
& \left.+\frac{1}{3} h^{2}\left[2 \bar{\zeta}_{1,1} \zeta_{1,1}+2 \bar{\zeta}_{2,1} \zeta_{2,1}+2\left(1-\frac{\varrho \ell_{2}^{2} \omega^{2}}{\pi^{2} \mu_{\kappa}}\right) \bar{\zeta}_{1,2} \zeta_{1,2}+\left(1-\frac{2 \varrho \ell_{2}^{2} \omega^{2}}{\pi^{2} \mu_{\kappa}}\right) \bar{\zeta}_{2,2} \zeta_{2,2}\right]\right\} d x_{2} .
\end{aligned}
$$

If $\varrho>0,(2-8)$ and (3-13) hold and

$$
\omega<\omega_{m} \quad \text { with } \quad \omega_{m}=\left[\frac{2 \varrho \ell_{2}^{2}}{\pi^{2} \mu_{\kappa}}\left(1+\frac{6 \ell_{2}^{2}}{\pi^{2} h^{2}}\right)\right]^{-1 / 2} .
$$

The frequency $\omega_{m}$ depends on the geometrical properties of the plate, namely $h$ and $\ell_{2}$. We can see that $\mu_{\kappa}$ is strictly positive and the critical frequency $\omega_{m}$ is such that

$$
0<1-\frac{\omega^{2}}{\omega_{m}^{2}}=\min \left\{1-\frac{2 \varrho \ell_{2}^{2} \omega^{2}}{\pi^{2} \mu_{\kappa}}\left(1+\frac{6 \ell_{2}^{2}}{\pi^{2} h^{2}}\right), 1-\frac{\varrho \ell_{2}^{2} \omega^{2}}{\pi^{2} \mu_{\kappa}}, 1-\frac{2 \varrho \ell_{2}^{2} \omega^{2}}{\pi^{2} \mu_{\kappa}}\right\} .
$$

Then, (4-15) and (4-17) lead to

$$
\begin{aligned}
\mathscr{g}_{\kappa}^{\prime}\left(x_{1}\right) \geq \int_{L_{x_{1}}} \mu_{\kappa}\left[p _ { 1 } ( \overline { \zeta } _ { 1 } + \overline { \psi } _ { , 1 } ) \left(\zeta_{1}\right.\right. & \left.+\psi_{, 1}\right)+p_{2} \bar{\psi}_{, 2} \psi, 2 \\
& \left.+\frac{1}{3} h^{2}\left(p_{3} \bar{\zeta}_{1,1} \zeta_{1,1}+p_{4} \bar{\zeta}_{2,1} \zeta_{2,1}+p_{5} \bar{\zeta}_{1,2} \zeta_{1,2}+p_{6} \bar{\zeta}_{2,2} \zeta_{2,2}\right)\right] d x_{2}
\end{aligned}
$$

where $p_{1}, \ldots, p_{6}$ represent the following strictly positive coefficients:

$$
p_{1}=p_{3}=p_{4}=2, \quad p_{2}=\frac{h^{2} \pi^{2}}{6 \ell_{2}^{2}+h^{2} \pi^{2}}\left(1-\frac{\omega^{2}}{\omega_{m}^{2}}\right), \quad p_{5}=2 p_{6}=2\left(1-\frac{\omega^{2}}{\omega_{m}^{2}}\right) .
$$

Under the above hypotheses, $\mathscr{F}_{\kappa}$ is a nondecreasing function and we can prove the following theorem.

Theorem 4.1. Let the hypotheses of Theorem 3.1 be still valid and let the frequency of harmonic vibrations $\omega$ be lower than the critical value $\omega_{m}$, as in (4-16). The function $\mathscr{F}_{\kappa}$ is such that

$$
\begin{aligned}
\left|\mathscr{g}_{\kappa}\left(x_{1}\right)\right| \leq \int_{L_{x_{1}}} \frac{\ell_{2}}{\pi}\left[P_{1}\left(\bar{\zeta}_{1}+\bar{\psi}_{, 1}\right)\left(\zeta_{1}+\psi, 1\right)+P_{2} \bar{\psi}_{, 2} \psi, 2\right. \\
\left.+\frac{1}{3} h^{2}\left(P_{3} \bar{\zeta}_{1,1} \bar{\zeta}_{1,1}+P_{4} \bar{\zeta}_{2,1} \zeta_{2,1}+P_{5} \bar{\zeta}_{1,2} \zeta_{1,2}+P_{6} \bar{\zeta}_{2,2} \zeta_{2,2}\right)\right] d x_{2}
\end{aligned}
$$


where

$$
P_{1}=c_{55}, \quad P_{2}=c_{55}, \quad P_{3}=c_{11}, \quad P_{4}=c_{66}, \quad P_{5}=\left(c_{11}+c_{66}+c_{12}\right), \quad P_{6}=\left(2 c_{66}+c_{12}\right) .
$$

Furthermore, $\mathscr{\Phi}_{\kappa}$ satisfies the inequality

$$
\mu_{\kappa}\left|\mathscr{F}_{k}\left(x_{1}\right)\right| \leq v \mathscr{F}_{k}^{\prime}\left(x_{1}\right) \quad \text { with } \quad v=\max _{i=1, \ldots, 6} \frac{\ell_{2} P_{i}}{\pi p_{i}}
$$

We underline that, as with $\omega_{m}, v$ also depends on the geometrical properties of the plate.

Proof. Considering (4-2)-(4-6), we arrive at

$\Phi_{\kappa}\left(x_{1}\right)=\int_{L_{x_{1}}} 2 \operatorname{Re}\left\{c_{55}\left(\bar{\zeta}_{1}+\bar{\psi}_{, 1}\right) \psi+\frac{1}{3} h^{2}\left[c_{11} \bar{\zeta}_{1,1} \zeta_{1}+\left(c_{66}-\kappa\right) \bar{\zeta}_{2,2} \zeta_{1}+\left(c_{12}+\kappa\right) \bar{\zeta}_{1,2 \zeta 2}+c_{66} \bar{\zeta}_{2,1} \zeta_{2}\right]\right\} d x_{2}$

Using the arithmetic-geometric and Schwarz inequalities and taking into account (4-10), we can show that

$$
\begin{aligned}
\int_{L_{x_{1}}} \operatorname{Re}\left[\left(\bar{\zeta}_{1}+\bar{\psi}_{, 1}\right) \psi\right] d x_{2} & \leq \frac{\ell_{2}}{\pi}\left(\int_{L_{x_{1}}}\left(\bar{\zeta}_{1}+\bar{\psi}_{, 1}\right)\left(\zeta_{1}+\psi_{, 1}\right) d x_{2}\right)^{1 / 2}\left(\int_{L_{x_{1}}} \bar{\psi}_{, 2} \psi, 2 d x_{2}\right)^{1 / 2} \\
& \leq \frac{\ell_{2}}{2 \pi}\left[\int_{L_{x_{1}}}\left(\bar{\zeta}_{1}+\bar{\psi}_{, 1}\right)\left(\zeta_{1}+\psi, 1\right) d x_{2}+\int_{L_{x_{1}}} \psi_{, 2} \bar{\psi}_{, 2} d x_{2}\right], \\
\int_{L_{x_{1}}} \operatorname{Re}\left[\bar{\zeta}_{1,1} \zeta_{1}\right] d x_{2} & \leq \frac{\ell_{2}}{\pi}\left(\int_{L_{x_{1}}} \bar{\zeta}_{1,1} \zeta_{1,1} d x_{2}\right)^{1 / 2}\left(\int_{L_{x_{1}}} \bar{\zeta}_{1,2} \zeta_{1,2} d x_{2}\right)^{1 / 2} \leq \frac{\ell_{2}}{2 \pi} \int_{L_{x_{1}}} \bar{\zeta}_{1, \alpha} \zeta_{1, \alpha} d x_{2}, \\
\int_{L_{x_{1}}} \operatorname{Re}\left[\bar{\zeta}_{2,2} \zeta_{1}\right] d x_{2} & \leq \frac{\ell_{2}}{\pi}\left(\int_{L_{x_{1}}} \bar{\zeta}_{2,2} \zeta_{2,2} d x_{2}\right)^{1 / 2}\left(\int_{L_{x_{1}}} \bar{\zeta}_{1,2} \zeta_{1,2} d x_{2}\right)^{1 / 2} \leq \frac{\ell_{2}}{2 \pi} \int_{L_{x_{1}}} \bar{\zeta}_{\alpha, 2} \zeta_{\alpha, 2} d x_{2}, \\
\int_{L_{x_{1}}} \operatorname{Re}\left[\bar{\zeta}_{1,2} \zeta_{2}\right] d x_{2} & \leq \frac{\ell_{2}}{\pi}\left(\int_{L_{x_{1}}} \bar{\zeta}_{1,2} \zeta_{1,2} d x_{2}\right)^{1 / 2}\left(\int_{L_{x_{1}}} \bar{\zeta}_{2,2} \zeta_{2,2} d x_{2}\right)^{1 / 2} \leq \frac{\ell_{2}}{2 \pi} \int_{L_{x_{1}}} \bar{\zeta}_{\alpha, 2} \zeta_{\alpha, 2} d x_{2}, \\
\int_{L_{x_{1}}} \operatorname{Re}\left[\bar{\zeta}_{2,1} \zeta_{2}\right] d x_{2} & \leq \frac{\ell_{2}}{\pi}\left(\int_{L_{x_{1}}} \bar{\zeta}_{2,1} \bar{\zeta}_{2,1} d x_{2}\right)^{1 / 2}\left(\int_{L_{x_{1}}} \bar{\zeta}_{2,2} \zeta_{2,2} d x_{2}\right)^{1 / 2} \leq \frac{\ell_{2}}{2 \pi} \int_{L_{x_{1}}} \bar{\zeta}_{2, \alpha} \zeta_{2, \alpha} d x_{2} .
\end{aligned}
$$

By means of (4-22) and (4-23), we obtain the inequality

$$
\begin{aligned}
\left|\mathscr{g}_{\kappa}\left(x_{1}\right)\right| \leq \int_{L_{x_{1}}} \frac{\ell_{2}}{\pi}\left\{c _ { 5 5 } \left(\bar{\zeta}_{1}\right.\right. & \left.+\bar{\psi}_{, 1}\right)\left(\zeta_{1}+\psi_{, 1}\right)+c_{55} \bar{\psi}_{, 2} \psi, 2 \\
& \left.+\frac{1}{3} h^{2}\left[c_{11} \bar{\zeta}_{1, \alpha} \zeta_{1, \alpha}+\left(c_{66}-\kappa\right) \bar{\zeta}_{\alpha, 2} \zeta_{\alpha, 2}+\left(c_{12}+\kappa\right) \bar{\zeta}_{\alpha, 2} \zeta_{\alpha, 2}+c_{66} \bar{\zeta}_{2, \alpha} \zeta_{2, \alpha}\right]\right\} d x_{2}
\end{aligned}
$$

which, through (4-20), leads to (4-19).

Since $\mu_{\kappa}$ and $p_{1}, \ldots, p_{6}$ are strictly positive, the definition of $v_{\kappa}$ and (4-19) allow us to write

$$
\begin{aligned}
\left|\mathscr{F}_{\kappa}\left(x_{1}\right)\right| \leq \int_{L_{x_{1}}} v\left[p _ { 1 } ( \overline { \zeta } _ { 1 } + \overline { \psi } _ { , 1 } ) \left(\zeta_{1}\right.\right. & +\psi, 1)+p_{2} \bar{\psi}_{, 2} \psi, 2 \\
& \left.+\frac{1}{3} h^{2}\left(p_{3} \bar{\zeta}_{1,1} \zeta_{1,1}+p_{4} \bar{\zeta}_{2,1} \zeta_{2,1}+p_{5} \bar{\zeta}_{1,2} \zeta_{1,2}+p_{6} \bar{\zeta}_{2,2} \zeta_{2,2}\right)\right] d x_{2} .
\end{aligned}
$$

Finally, (4-18) and (4-24) prove that (4-21) is satisfied. 
For what follows, it is useful to introduce the class of steady-state vibrations $\left(\zeta_{\alpha}, \psi\right)$ for which

$$
\begin{aligned}
\mathscr{E}\left(x_{1}\right)=\int_{S_{x_{1}}} \frac{\ell_{2}}{\pi}\left[P_{1}\left(\bar{\zeta}_{1}+\bar{\psi}_{, 1}\right)\left(\zeta_{1}+\psi_{, 1}\right)+P_{2} \psi_{, 2} \bar{\psi}_{, 2}\right. \\
\left.+\frac{1}{3} h^{2}\left(P_{3} \zeta_{1,1} \bar{\zeta}_{1,1}+P_{4} \bar{\zeta}_{2,1} \zeta_{2,1}+P_{5} \bar{\zeta}_{1,2} \zeta_{1,2}+P_{6} \bar{\zeta}_{2,2} \zeta_{2,2}\right)\right] d a<\infty,
\end{aligned}
$$

where $S_{x_{1}}=\bar{\Sigma} \cap\left[x_{1}, \infty\right) \times\left[0, \ell_{2}\right]$. From a direct differentiation of $\mathscr{E}\left(x_{1}\right)$, we get

$$
\begin{aligned}
-\mathscr{E}^{\prime}\left(x_{1}\right)=\int_{L_{x_{1}}} \frac{\ell_{2}}{\pi}\left[P_{1}\left(\bar{\zeta}_{1}+\bar{\psi}_{, 1}\right)\left(\zeta_{1}+\psi, 1\right)+P_{2} \psi, 2 \bar{\psi}_{, 2}\right. \\
\left.+\frac{1}{3} h^{2}\left(P_{3} \zeta_{1,1} \bar{\zeta}_{1,1}+P_{4} \zeta_{2,1} \bar{\zeta}_{2,1}+P_{5} \zeta_{1,2} \bar{\zeta}_{1,2}+P_{6} \zeta_{2,2} \bar{\zeta}_{2,2}\right)\right] d x_{2} .
\end{aligned}
$$

As a consequence of (4-19) and (4-26), it follows that

$$
\left|\mathscr{E}_{\kappa}\left(x_{1}\right)\right| \leq-\mathscr{E}^{\prime}\left(x_{1}\right)
$$

so $\mathscr{E}\left(x_{1}\right)$ is a nonincreasing function.

When the strip $\bar{\Sigma}$ is bounded, the hypothesis (4-25) is trivially satisfied; Equations (4-5) and (4-27) imply

$$
\mathscr{E}^{\prime}\left(\ell_{1}\right)=0 \Rightarrow \mathscr{F}_{\kappa}\left(\ell_{1}\right)=0 \text {. }
$$

On the other side, if the strip $\bar{\Sigma}$ is semiinfinite and the hypothesis (4-25) is satisfied, then (4-26) and (4-27) lead to

$$
\lim _{x_{1} \rightarrow \infty} \mathscr{E}^{\prime}\left(x_{1}\right)=0 \Rightarrow \lim _{x_{1} \rightarrow \infty} \mathscr{J}_{\kappa}\left(x_{1}\right)=0
$$

Now, provided that the frequency of harmonic vibrations is lower than the critical value $\omega_{m}$, we can establish the following theorem.

Theorem 4.2. Let the hypotheses of Theorem 4.1 be still valid and let $\left\{\zeta_{\alpha}, \psi\right\}$ be the steady-state vibrations for which (4-25) holds. Then, the function $\mathscr{\Phi}_{\kappa}$ satisfies a decay estimate of Saint-Venant type:

$$
0 \leq-\mathscr{g}_{\kappa}\left(x_{1}\right) \leq-\mathscr{g}_{\kappa}(0) \exp \left(-\frac{\mu_{\kappa}}{v} x_{1}\right) \text {. }
$$

Proof. Whether the considered strip is bounded or not, from hypothesis (4-16) on the frequency $\omega$ and from the nondecreasing property of $\mathscr{F}_{\kappa},(4-28)$ and (4-29) imply

$$
\mathscr{g}_{\kappa}\left(x_{1}\right) \leq 0 .
$$

We can also remark, from the definitions of coefficients $P_{1}, \ldots, P_{6}$ and $v$, that $v$ is strictly positive. Then, (4-21) and (4-31) allow us to write

$$
\mathscr{E}_{\kappa}^{\prime}\left(x_{1}\right)+\frac{\mu_{\kappa}}{v} \mathscr{F}_{\kappa}\left(x_{1}\right) \geq 0 \Rightarrow \frac{d}{d x_{1}}\left[\mathscr{F}_{\kappa}\left(x_{1}\right) \exp \left(\frac{\mu_{\kappa}}{v} x_{1}\right)\right] \geq 0 .
$$

It is easy to observe that (4-32) leads to the estimate (4-30) with exponential decay factor $\mu_{\kappa} / \nu$.

Remark. The investigation performed is based on an assumption concerning the strong ellipticity of the elasticity tensor. The results obtained under such an hypothesis are thus valid also for classes of particular materials characterized by special properties, like negative Poisson's ratio and negative stiffness (auxetic or antirubber materials). These particular structures (see, for example, [Park and Lakes 2007]) expand laterally when stretched, in contrast to ordinary materials. 


\section{List of symbols}

Right cylinder of height $2 h$

Symbol Page

Interiors of $\bar{B}$ and $\bar{\Sigma}$

$\bar{B}$

325

Rectangular Cartesian coordinate frame

$B, \Sigma$

325

Variable functions characterizing a bending state

$O x_{1} x_{2}$

325

Constitutive equations

$v_{\alpha}, w$

325

Family of tensors depending on a parameter $\kappa$

$M_{i j}, \tau_{i j}$

325

Definition of initial-boundary value problem

$\tilde{M}_{i j}$

External data

$\mathscr{P}$

Support of external data

$\mathscr{D}$

Set defined on the plate

$\hat{D}_{T}$

Integral function

$D_{r}, \Sigma_{r}, L_{r}$

Quadratic forms

$\Phi_{\kappa}$

Matrices associated with the quadratic forms $\mathscr{W}_{0}, \mathscr{W}_{1}, \mathscr{W}_{2}$

$\mathscr{T}, W_{,}, W_{0}, W_{1}, W_{2}$

Smallest and largest eigenvalues of $\mathscr{A}_{0}, \mathscr{A}_{1}, \mathscr{A}_{2}$

$\mathscr{A}_{0}, \mathscr{A}_{1}, \mathscr{A}_{2}$

328

Smallest and largest eigenvalues of $\mathcal{W}_{\alpha}$

Constant characteristic of the material

$\mu_{\kappa}, \eta_{\kappa}$

$k_{m}^{(\alpha)}, k_{M}^{(\alpha)}$

$c_{\kappa}$

Variable functions characterizing steady-state vibrations

$\zeta_{\alpha}, \psi$

332

Frequency of oscillations

$\omega$

332

Dimensions of the middle section of the plate

$\ell_{1}, \ell_{2}$

332

Constitutive equations for problem of steady-state vibrations

$\Gamma_{i j}, \chi_{i}$

Set defined on the plate for steady-state vibrations

$L_{x_{1}}$

Integral function for steady-state vibrations

Complex quadratic forms

$\mathscr{F}_{\kappa}$

Estimate coefficients

$\widehat{\mathscr{W}}, \widehat{\mathscr{W}}_{0}, \widehat{\mathscr{W}}_{1}, \widehat{\mathscr{W}}_{2}$

Estimate coefficients

Constant characteristic of the material

$p_{1}, \ldots, p_{6}$

$P_{1}, \ldots, P_{6}$

Integral function

$v$

Set defined on the plate for steady-state vibrations

$\mathscr{E}$

336

$S_{x_{1}}$

\section{References}

[Arnold et al. 2002] D. N. Arnold, A. L. Madureira, and S. Zhang, "On the range of applicability of the Reissner-Mindlin and Kirchhoff-Love plate bending models", J. Elasticity 67:3 (2002), 171-185.

[Chiriță 1995] S. Chiriță, "Spatial decay estimates for solutions describing harmonic vibrations in a thermoelastic cylinder", $J$. Therm. Stresses 18:4 (1995), 421-436.

[Chiriță and Ciarletta 1999] S. Chiriță and M. Ciarletta, "Time-weighted surface power function method for the study of spatial behaviour in dynamics of continua", Eur. J. Mech. A Solids 18:5 (1999), 915-933.

[Chiriță and Ciarletta 2003] S. Chiriță and M. Ciarletta, "Some further growth and decay results in linear thermoelastodynamics", J. Therm. Stresses 26:9 (2003), 889-903. 
[Chiriță et al. 2007] S. Chiriță, A. Danescu, and M. Ciarletta, "On the strong ellipticity of the anisotropic linearly elastic materials", J. Elasticity 87:1 (2007), 1-27.

[Ciarletta et al. 2005] M. Ciarletta, S. Chiriță, and F. Passarella, "Some results on the spatial behaviour in linear porous elasticity", Arch. Mech. 57:1 (2005), 43-65.

[Constanda 1990] C. Constanda, A mathematical analysis of bending of thin plates with transverse shear deformation, Longman, Essex, 1990.

[Fabrizio and Chiriță 2004] M. Fabrizio and S. Chiriță, "Some qualitative results on the dynamic viscoelasticity of the ReissnerMindlin plate model”, Q. J. Mech. Appl. Math. 57:1 (2004), 59-78.

[Flavin 1974] J. N. Flavin, “On Knowles' version of Saint-Venant's principle in two-dimensional elastostatics", Arch. Ration. Mech. An. 53:4 (1974), 366-375.

[Green and Naghdi 1967] A. E. Green and P. M. Naghdi, "The linear theory of an elastic Cosserat plate", Math. Proc. Cambridge Philos. Soc. 63 (1967), 537-550.

[Gregory and Wan 1985] R. D. Gregory and F. Y. M. Wan, "On plate theories and Saint-Venant's principle", Int. J. Solids Struct. 21:10 (1985), 1005-1024.

[Gurtin 1972] M. E. Gurtin, Linear theory of elasticity, edited by S. Flügge, Handbuch der Physik VIa/2, Springer, Berlin, 1972.

[Horgan 1989] C. O. Horgan, "Recent developments concerning Saint-Venant's principle: an update", Appl. Mech. Rev. (ASME) 42:11 (1989), 295-303.

[Horgan 1996] C. O. Horgan, "Recent developments concerning Saint-Venant's principle: a second update", Appl. Mech. Rev. (ASME) 49:10S (1996), S101-S111.

[Horgan and Knowles 1983] C. O. Horgan and J. K. Knowles, "Recent developments concerning Saint-Venant's principle", Adv. Appl. Mech. 23 (1983), 179-269.

[Hughes 1987] T. J. R. Hughes, The finite element method: linear static and dynamic finite element analysis, Prentice-Hall, Englewood Cliffs, NJ, 1987.

[Knowles 1966] J. K. Knowles, "On Saint-Venant's principle in the two-dimensional linear theory of elasticity", Arch. Ration. Mech. An. 21:1 (1966), 1-22.

[Lagnese and Lions 1988] J. E. Lagnese and J. L. Lions, Modelling, analysis and control of thin plates, Collection RMA 6, Masson, Paris, 1988.

[Mielke 1988] A. Mielke, “On Saint-Venant's problem for an elastic strip”, Proc. R. Soc. Edinburgh A Math. 110:3 (1988), $161-181$.

[Mindlin 1951] R. D. Mindlin, "Influence of rotatory inertia and shear on flexural motions of isotropic, elastic plates", J. Appl. Mech. (ASME) 18:1 (1951), 31-38.

[Naghdi 1971] P. M. Naghdi, The theory of shells and plates, edited by C. Truesdell, Handbuch der Physik VIa/2, Springer, Berlin, 1971.

[Nowinski 1978] J. L. Nowinski, Theory of thermoelasticity with applications, Sijthoff \& Noordoff, Alphen aan den Rijn, 1978.

[Park and Lakes 2007] J. B. Park and R. S. Lakes, Biomaterials, 3rd ed., Springer, Berlin, 2007.

[Paroni et al. 2006] R. Paroni, P. Podio-Guidugli, and G. Tomassetti, "The Reissner-Mindlin plate theory via $\Gamma$-convergence", C. R. Acad. Sci. Paris Sér. I Math. 343:6 (2006), 437-440.

[Passarella and Zampoli 2009a] F. Passarella and V. Zampoli, "Some results concerning the state of bending for transversely isotropic plates", Math. Methods Appl. Sci. 32:14 (2009), 1828-1843.

[Passarella and Zampoli 2009b] F. Passarella and V. Zampoli, "Spatial estimates for transient and steady-state solutions in transversely isotropic plates of Mindlin-type", Eur. J. Mech. A Solids 28:4 (2009), 868-876.

[Reissner 1945] E. Reissner, "The effect of transverse shear deformation on the bending of elastic plates", J. Appl. Mech. (ASME) 12:2 (1945), 68-77.

[Reissner 1947] E. Reissner, “On bending of elastic plates”, Quart. Appl. Math. 5:1 (1947), 55-68. 
[Schiavone and Tait 1993] P. Schiavone and R. J. Tait, "Thermal effects in Mindlin-type plates", Q. J. Mech. Appl. Math. 46:1 (1993), 27-39.

[Toupin 1965] R. A. Toupin, "Saint-Venant's principle”, Arch. Ration. Mech. An. 18:2 (1965), 83-96.

[Wheeler and Sternberg 1968] L. T. Wheeler and E. Sternberg, "Some theorems in classical elastodynamics", Arch. Ration. Mech. An. 31:1 (1968), 51-90.

Received 2 Mar 2009. Revised 25 Sep 2009. Accepted 30 Sep 2009.

FRANCESCA PASSARELla: passarella@diima.unisa.it

Department of Information Engineering and Applied Mathematics, University of Salerno, via Ponte Don Melillo, 84084 Fisciano (SA), Italy

Vincenzo Tibullo: vtibullo@unisa.it

Department of Information Engineering and Applied Mathematics, University of Salerno, via Ponte Don Melillo, 84084 Fisciano (SA), Italy

VITTORIO ZAMPOLI: vzampoli@unisa.it

Department of Information Engineering and Applied Mathematics, University of Salerno, via Ponte Don Melillo, 84084 Fisciano (SA), Italy 


\title{
JOURNAL OF MECHANICS OF MATERIALS AND STRUCTURES
}

\author{
http://www.jomms.org
}

\author{
Founded by Charles R. Steele and Marie-Louise Steele

\section{EDITORS} \\ Charles R. STEele \\ DAVIDE BIGONI \\ IWONA JASIUK \\ YASUHIDE SHINDO \\ Stanford University, U.S.A. \\ University of Trento, Italy \\ University of Illinois at Urbana-Champaign, U.S.A. \\ Tohoku University, Japan
}

\section{EDITORIAL BOARD}

H. D. BUI École Polytechnique, France

J. P. CARTER University of Sydney, Australia

R. M. Christensen Stanford University, U.S.A.

G. M. L. GLADWELL University of Waterloo, Canada

D. H. HodGES Georgia Institute of Technology, U.S.A.

J. HUTCHINSON Harvard University, U.S.A.

C. HwU National Cheng Kung University, R.O. China

B. L. KariHaloo University of Wales, U.K.

Y. Y. KIM Seoul National University, Republic of Korea

Z. Mroz Academy of Science, Poland

D. PAMPlonA Universidade Católica do Rio de Janeiro, Brazil

M. B. RUBIN Technion, Haifa, Israel

A. N. SHUPIKov Ukrainian Academy of Sciences, Ukraine

T. TARNAI University Budapest, Hungary

F. Y. M. WAN University of California, Irvine, U.S.A.

P. WRIGGERS Universität Hannover, Germany

W. YANG Tsinghua University, P.R. China

F. ZIEGLER Technische Universität Wien, Austria

\section{PRODUCTION}

\section{Paulo Ney de Souza Production Manager \\ SheIla Newbery Senior Production Editor \\ SILVIO LEVY Scientific Editor}

See inside back cover or http://www.jomms.org for submission guidelines.

JoMMS (ISSN 1559-3959) is published in 10 issues a year. The subscription price for 2010 is US $\$ 500 /$ year for the electronic version, and \$660/year (+\$60 shipping outside the US) for print and electronic. Subscriptions, requests for back issues, and changes of address should be sent to Mathematical Sciences Publishers, Department of Mathematics, University of California, Berkeley, CA 94720-3840.

JoMMS peer-review and production is managed by EditFLOW ${ }^{\mathrm{TM}}$ from Mathematical Sciences Publishers.

PUBLISHED BY

mathematical sciences publishers

http://www.mathscipub.org

A NON-PROFIT CORPORATION

Typeset in LATEX

CCopyright 2010. Journal of Mechanics of Materials and Structures. All rights reserved. 


\title{
Journal of Mechanics of Materials and Structures
}

\author{
Volume 5, No. 2 February 2010
}

A critical analysis of interface constitutive models for the simulation of delamination in composites and failure of adhesive bonds

Anton Matzenmiller, Sebastian Gerlach and Mark Fiolka

185

Computational studies of collagen fibril biominerals using a virtual internal bond

model with extrinsic length scale

Ganesh Thiagarajan and Kavita Deshmukh

The simulation of stochastically excited viscoelastic systems and their stability

VADIM D. POTAPOV

Fundamental solutions for an inhomogeneous cross-anisotropic material due to horizontal and vertical plane strain line loads

Cheng-Der Wang, Jia-YAN Hou and WeI-Jer WANG

Mechanical and fracture analysis of welded pearlitic rail steels

Aldinton Allie, Heshmat A. Aglan and Mahmood Fateh

Rate dependence of indentation size effects in filled silicone rubber

Ramanjaneyulu V. S. TATIRAJU and ChUng-SOUK HAN

A novel application of a laser Doppler vibrometer in a hèalth monitoring system

DAVOOD REZAEI and FARID TAHERI

Energy absorption of a helicoidal bistable structure

Seubpong Leelavanichkul, Andrej Cherkaev, Daniel O. Adams

and FLORIAN SOLZBACHER

Decay properties of solutions of a Mindlin-type plate model for rhombic systems

Francesca Passarella, Vincenzo Tibullo and VitTorio Zampoli

A consistent refinement of first-order shear deformation theory for laminated composite and sandwich plates using improved zigzag kinematics Alexander Tessler, Marco Di Sciuva and Marco Gherlone 